\title{
Gastrointestinal and Liver Disorders Encountered during the Covid-19-Pandemic-Strategies to Overcome and Nutritional Input to Boost Immunity
}

\author{
Kulvinder Kochar Kaur ${ }^{1 *}$, Gautam Allahbadia ${ }^{2}$ and Mandeep Singh ${ }^{3}$ \\ ${ }^{1}$ Scientific Director, DR Kulvinder Kaur Centre for Human Reproduction, Jalandhar, \\ Punjab, India \\ ${ }^{2}$ Scientific Director, Rotunda-A Centre for Human Reproduction, Mumbai, India \\ ${ }^{3}$ Consultant Neurologist, Swami Satyanand Hospital, Jalandhar, Punjab, India \\ *Corresponding Author: Kulvinder Kochar Kaur, Scientific Director, DR Kulvinder \\ Kaur Centre for Human Reproduction, Jalandhar, Punjab, India.
}

Received: July 25, 2020

Published: September 16, 2020

(C) All rights are reserved by Kulvinder

Kochar Kaur., et al.
We had reviewed on various aspects of COVID-19-disease from structure, presentation management, pregnancy and lactation, management of severe COVID-19-disease and effects on reproductive system [1-5]. Here we have emphasized on the GIT involvement aspect, besides liver and implications on endoscopy practice worldwide in, COVID-19 era. Digestive symptoms including anorexia, nausea, vomiting, diarrhea $(\mathrm{N}, \mathrm{V}, \mathrm{D})$ - frequently reported in patients with COVID-19-disease [CD]. SARS-CoV-2 RNA was first detected in stool of the $1^{\text {st }}$ reported CD-19 case in the USA, who also presented with the digestive symptoms of $(\mathrm{N}, \mathrm{V}, \mathrm{D})$. In the biggest cohort-1099 patients with laboratory-confirmed CD-19 from 552 hospitals in China in January 2020, N, V, D were reported in $55(5 \%)$ and $42(3.8 \%)$ patients, respectively. Of the 204 patients with CD-19 and full investigations, 99 (48.5\%) presented with digestive symptoms as main complaint. Patients with digestive symptoms had separate manifestations, like anorexia (83.8\%), V, $\mathrm{D}$ and abdominal pain (0.4\%). Digestive symptoms - might be presenting prior to respiratory symptoms, or only symptoms of CD19. The mode for GIT infection of SARS-CoV is ACE2 cell receptor. SARS-CoV-2, having genome sequence of SARS-CoV (82\%), might use the same ACE2, strain -SARS-CoV (2003). Xiao., et al. showed on endoscopic biopsy samples that ACE2 was rarely expressed in esophageal epithelium, but abundantly distributed in cilia of glandular epithelia, while staining of viral nucleocapsid protein was visualized in the cytoplasm of gastric, duodenal and rectum glandular epithelial cell, but not in esophageal epithelium. As found in another study in the small intestine, the crosstalk among SARSCoV-2-ACE2 might disrupt the function of ACE2 and cause diarrhea. Chances of fecal-oral transmission of SARS-CoV-2 stressed on proper hand hygiene, stools handling and hospital sewage carefully in patients. SARS-CoV-2 in the GIT, signifies CD-19 infection in patients with preexisting digestive diseases and fecal microbiota transplant donors. To prevent SARS-CoV-2 transmission by fecal microbiota transplantation, more testing needed over current ones. GIT endoscopy departments face marked transmissions risk of virus during endoscopy. Earliest reports of CD-19, 40/138 showed high risk of infection for healthcare workers of infection. Possible routes of viral transmission on endoscopy examination - personto-person, respiratory droplets, aerosols formed on endoscopy and contact with contaminated surroundings, body fluids and fecal material. Recommendation used-World Endoscopy Organization, ASGE, ESGE on endoscopy during the pandemic. Liver damage was common in the patients infected by the other 2 highly pathogenic coronavirus-SARS-CoV, MERS CoV associated with the severity of diseases. In patients with CD-19, several studies have documented the incidence of liver injury, indicating that $2-11 \%$ of patients with CD-19 had liver comorbidities and 16 - 53\% cases reported abnormal levels of SGOT/PT. Guan., et al. and Huang showed that elevated AST levels were observed more in patients with severe disease. Liver injury might be due to viral infection in liver cells or due to other causes such as drug induced liver injury and systemic inflammation induced by CKS or pneumonia-related hypoxia. SARS virus has been shown to be present in the liver tissue, although the viral titer was relatively low because viral inclusions were not observed. The impact of COVID-19 in patients with preexisting chronic liver diseases, needs evaluation. From Chinese study showed that chronic hepatitis B infection patients didn't have higher disease severity vs population as did world cases. Major changes to the standard

Citation: Kulvinder Kochar Kaur., et al. "Gastrointestinal and Liver Disorders Encountered during the Covid-19-Pandemic-Strategies to Overcome and Nutritional Input to Boost Immunity". Acta Scientific Microbiology 3.10 (2020): 64-67. 
management of GIT diseases, priorities like minimizing viral transmission, preserving PPE and freeing hospital beds have driven unconventional approaches to managing GI patients. Conversion of endoscopy units to CD units and redeployment of GI fellows and faculty has profoundly changed most GI services. Meanwhile, consult and procedural volumes have reduced drastically [7]. Sethi., et al. [8] showed how dealing specific consults and conditions has changed, the main focus is on noninvasive measures and maximizing medical therapies. Endoscopic procedures have been reserved for those timely interventions that are most likely to be therapeutic. The role of multidisciplinary discussion, although important, has become critical now. Advancing a clear vision and a transparent process for how to organize and triage care in the recovery phase will allow for a smooth transition to our new normal [8]. For percutaneous endoscopic gastrostomy (PEG) placements even in this time of pandemic needs to be avoided. Despite the viral effects of the SARS-CoV-2 in the GIT, enteral nutrition is still the preferred method of nutrition therapy for patients with CD-19 because of its multiple benefits in the gut. It supports the structural and functional integrity of the gut, thereby modulating systemic immunity, attenuating disease severity, aiding in good results. However, the presence of GI symptoms such as anorexia, N, V, D in some patients with CD-19 complicates this challenge to feed the patient [9].

For emergency colorectal surgery, like cancer surgery, queries on operating room (OR) utilization and techniques needed to be addressed. Caregiver safety relating to PPE and OR environment. With, extra PPE may be required on local presence of CD-19 testing plus incidence of known infection there. Besides standard CD19 PPE precautions, a negative-pressure environment, including an $\mathrm{OR}$, is advocated, mainly for aerosol-generating procedures (AGPs). Hospital spaces from patient wards to ORs to endoscopy rooms have been successfully converted from standard positivepressure to negative-pressure spaces. Moreover, minimally invasive surgery with pneumoperitoneum is an AGP and thus must be carefully considered. Present worry is to avoid in patients infected with SARS-CoV-2 or we can do under precautions with safety measures in place -minimize exposure to aerosolized virus particles. Significant lessons learned from pressurized intraperitoneal aerosolized chemotherapy procedures are helpful to improve our insight and treat [10]. Mayo Clinic researchers, used a computer model to compare three strategies for CD-19 testing using PCR for patients scheduled to undergo endoscopic procedures during the
CD-19 pandemic: strategy 1: testing all patients within 48 hours, with urgent procedures performed regardless of PCR result using high-risk PPE, and semi-urgent procedures performed with a negative result using low-risk PPE; $2^{\text {nd: }}$ testing for semi-urgent procedures; and $3^{\text {rd }}$ endoscopy for urgent procedures only.

PCR testing was safe and effective intervention to resume endoscopy in semi-urgent and elective cases, requiring expenditures US22 - \$105 in testing per patient for strategies 2 and 3 respectively. This would allow an increase to $19.4 \%$ and $95.3 \%$ of baseline endoscopies, respectively. Implementing nationwide PCR testing in the US would require 13 million to $\$ 64$ million per week for the two strategies, respectively, with a return of $\$ 165$ million and $\$ 767$ million to providers, respectively. Healthcare workers infected in the endoscopy unit weekly were around 65 and 325 for the two strategies, respectively. Per Aytin., et al. points only life-threatening emergency operations be performed in the stage where the epidemic exceeds the capacity of the hospitals ( $1^{\text {st }}$ stage), cancer and transplantation surgery should be initiated when the outbreak begins to be controlled ( $2^{\text {nd }}$ stage), and surgery for elective cases be performed in a controlled manner with suppression of the outbreak ( $3^{\text {rd }}$ stage). Most cases can be postponed to the $3^{\text {rd }}$ stage of the epidemic [11-15].

Challenges arise concerning how to optimally support the immune systemin the general population, especially under self-confinement. An optimal immune response depends on an adequate diet and nutrition in order to keep infection at bay. For example, sufficient protein intake is crucial for optimal antibody production. Low micronutrient status, such as of vitamin A or zinc, has been associated with increased infection risk. Frequently, poor nutrient status is associated with inflammation and oxidative stress, which in turn can impact the immune system. Dietary constituents with especially high anti-inflammatory and antioxidant capacity include vitamin C, vitamin E and phytochemicals such as carotenoids and polyphenols. Several of these can interact with transcription factors such as NF-kB and Nrf-2, related to anti-inflammatory and antioxidant effects, respectively. Vitamin D in particular may perturb viral cellular infection via interacting with cell entry receptors (angiotensin converting enzyme 2), ACE2. Dietary fiber, fermented by the gut microbiota into short-chain fatty acids, has also been shown to produce anti-inflammatory effects. 
In this short communication, we highlight the importance of an optimal status of relevant nutrients to effectively reduce inflammation and oxidative stress, thereby strengthening the immune system during the COVID-19 crisis (Figure 2 regarding diet and other prophylaxis) [16] and figure 3 on potential targets for COVID-19 disease therapy [17].

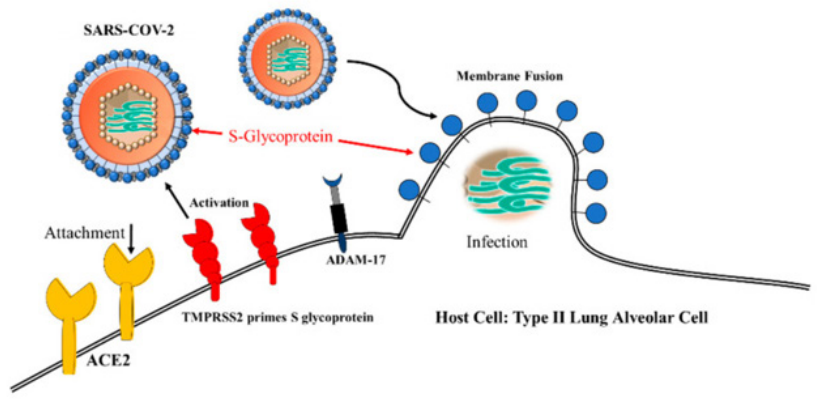

Figure 1: Courtesy ref no-6. SARS-CoV-2 uses angiotensin-converting enzyme 2 (ACE2) for cell entry through TMPRSS2 priming of S glycoprotein.

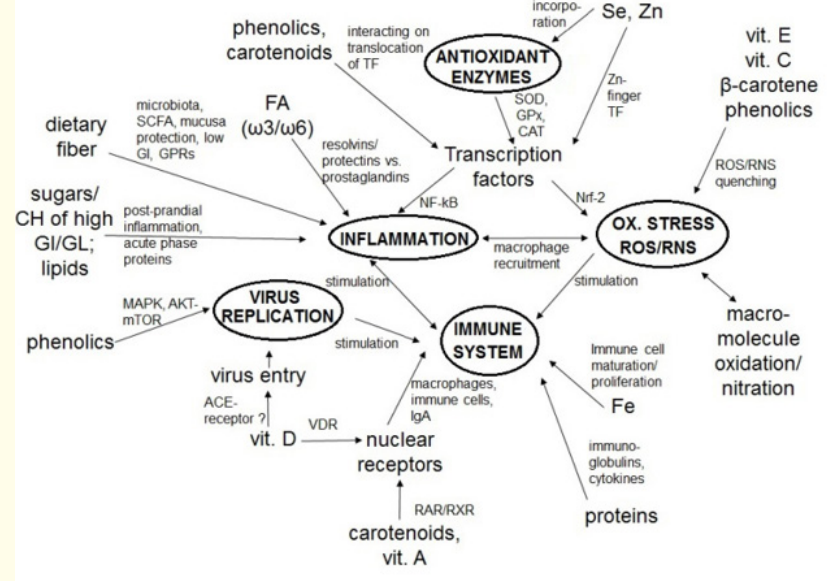

Figure 2: Courtesy ref no-16. Schematic diagram showing interactions between selected dietary constituents, the immune system, and viral infection. Abbreviations: $\mathrm{CH}$ : Carbohydrates; GALT: Gut-Associated Lymphoid Tissue; GPRs: G-Protein-Coupled Receptors; FA: Fatty Acids; GI/GL: Glycemic Index/Load; RAR/ RXR: Retinoic Acid Receptor/Retinoid X Receptor; SCFA: ShortChain Fatty Acids; TF: Transcription Factors; VDR: Vitamin D Receptor.

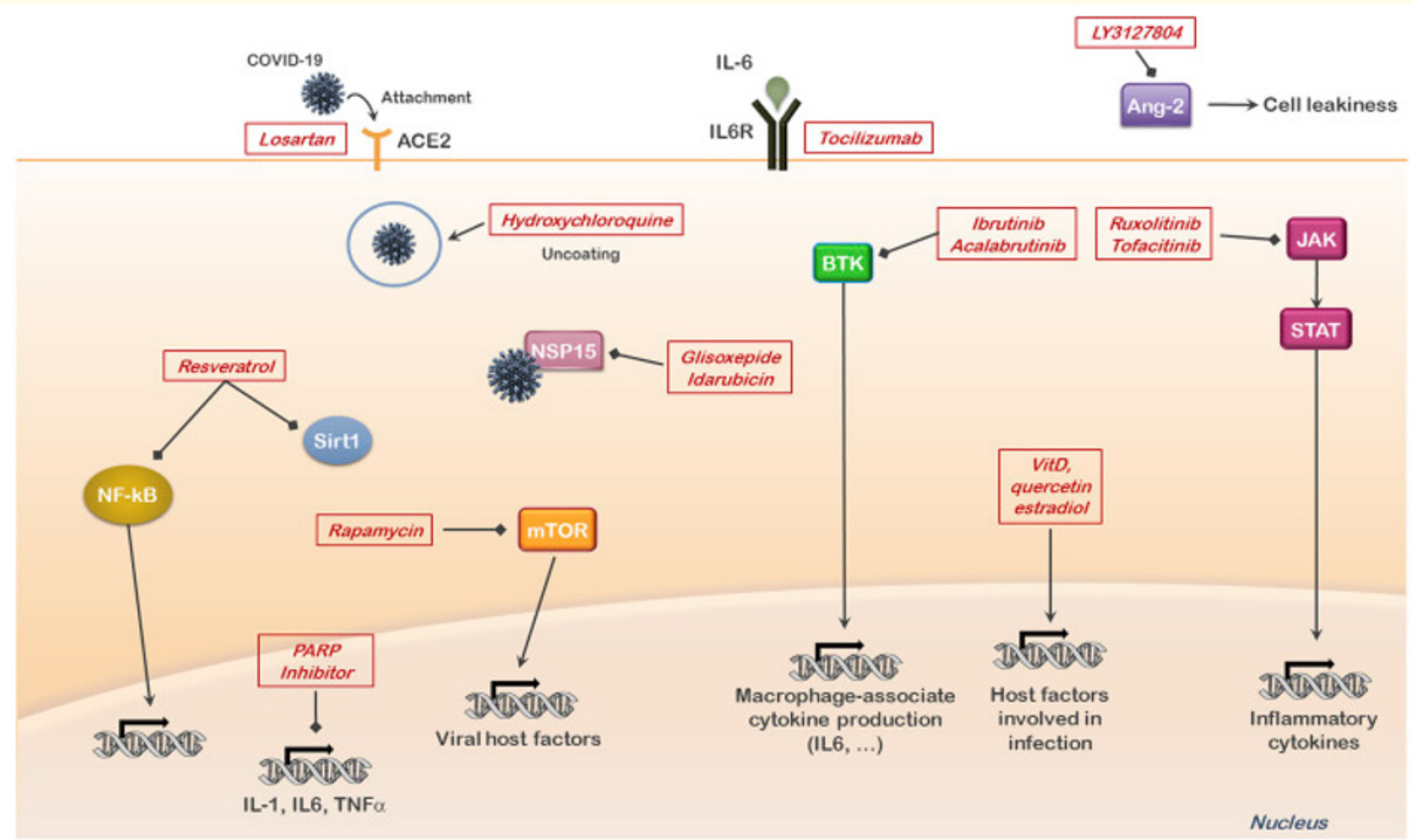

Figure 3: Courtesy ref no-17. Overview of potential sites of therapeutic intervention in COVID-19 disease. 


\section{Bibliography}

1. Kulvinder Kochar Kaur., et al. "A Comprehensive review on epidemiology, aetiopathogenesis, diagnosis and treatment of the novel corona virus syndrome -COVID-19". Iberoamerican Journal of Medicine 2 (2020): 110-123.

2. Kulvinder Kochar Kaur. "Editorial -Maximum possible efforts by which we will win over this Deadly Pandemic of COVID-19". Acta Scientific Microbiology 3.6 (2020): 01-03.

3. Kulvinder Kochar Kaur., et al. "An Update on COVID19 from Etiopathogenesis, to role in male infertility". Pregnancy Lactation, and Regarding treatment (2020).

4. Kulvinder Kochar Kaur., et al. "Editorial-An update on management of severe COVID19presenting with Cytokine Release Syndrome responsible for most mortalities in COVID19". Acta Scientific Microbiology 3.8 (2020): 01-06.

5. Kulvinder Kochar Kaur., et al. "An update on the place of ART with SARS CoV2-around and influence on Both Reproduction, transmission possibilities to, Progeny, Partners and Staff - What is the Answer". Under review with Longdom Publishers.

6. Kim Cho. "SARS-CoV-2 Evolutionary Adaptation toward Host Entry and Recognition of Receptor 0-AcetylSialylation in Virus-Host Interaction". International Journal of Molecular Sciences 21.12 (2020): 4549.

7. Aguila EJT., et al. "COVID-19 and its effects on the digestive system and endoscopy practice". Journal of Gastroenterology and Hepatology 4 (2020) 324-331.

8. Sethi A., et al. "Donning a New Approach to the Practice of Gastroenterology: Perspectives From the COVID-19 Pandemic Epicenter". Clinical Gastroenterology and Hepatology 18 (2020): 1673-1681.

9. Aguila EJT., et al. "Follow Your Gut: Challenges in Nutritional Therapy During the COVID-19 Pandemic". Clinical Gastroenterology and Hepatology (2020).

10. Soetikno R., et al. "Considerations in performing endoscopy during the COVID-19 pandemic". Gastrointestinal Endoscopy 92.1 (2020): 176-183.

11. Montorfano L., et al. "Colorectal surgery obesity-related morbidity during COVID-19. Surgery for Obesity and Related Diseases". Surgery for Obesity and Related Diseases (2020): 1-4.

12. Corral JE., et al. "COVID-19 polymerase chain reaction testing before endoscopy: an economic analysis". Gastrointestinal Endoscopy (2020): 1-11.

13. Bilal M., et al. "What constitutes urgent endoscopy? A social media snapshot of gastroenterologists' views during the COVID-19 pandemic". Endoscopy International Open 8.5 (2020): E693-E698.
14. Sawhney MS., et al. "Triaging advanced GI endoscopy procedures during the COVID-19 pandemic: consensus recommendations using the Delphi method". Gastrointestinal Endoscopy (2020).

15. Agyun N., et al. "Endocrine Surgery during the COVID-19 Pandemic: Recommendations from the Turkish Association of Endocrine Surgery". The Medical Bulletin of Sisli Etfal Hospital 54.2 (2020): 113-117.

16. Iddir M., et al. "Strengthening the Immune System and Reducing Inflammation and Oxidative Stress through Diet and Nutrition: Considerations during the, COVID-19 Crisis". Nutrients 12 (2020): 1562.

17. Akula SM., et al. "Cancer therapy and treatments during COVID-19 era". Advances in Biological Regulation 77 (2020): 100739.

\section{Assets from publication with us}

- Prompt Acknowledgement after receiving the article

- Thorough Double blinded peer review

- Rapid Publication

- Issue of Publication Certificate

- High visibility of your Published work

Website: https://www.actascientific.com/

Submit Article: https://www.actascientific.com/submission.php Email us: editor@actascientific.com

Contact us: +919182824667 